\title{
À quoi servent les recherches en éducation?
}

Introduction

What is education research used for? Introduction

¿De qué sirven las investigaciones en educación? Introducción

Jean-Marie De Ketele

\section{OpenEdition}

\section{Journals}

Édition électronique

URL : https://journals.openedition.org/ries/10027

DOI : $10.4000 /$ ries. 10027

ISSN : 2261-4265

\section{Éditeur}

France Education international

\section{Édition imprimée}

Date de publication : 1 décembre 2020

Pagination : 51-64

ISBN : 978-2-85420-628-9

ISSN : $1254-4590$

Référence électronique

Jean-Marie De Ketele, "À quoi servent les recherches en éducation? », Revue internationale d'éducation de Sèvres [En ligne], 85 I décembre 2020, mis en ligne le 01 décembre 2020, consulté le 07 décembre 2022. URL : http://journals.openedition.org/ries/10027 ; DOI : https://doi.org/10.4000/ries. 10027 


\title{
La recherche en éducation
}

\section{Introduction}

\section{À quoi servent les recherches en éducation?}

\author{
Jean-Marie De Ketele \\ Université catholique de Louvain
}

Largement répercutée et amplifiée par les médias, la crise de la Covid-19 a fait découvrir l'importance des chercheurs en virologie et en épidémiologie dans les décisions prises pour juguler la pandémie. Elle a montré aussi les limites de l'état actuel de nos connaissances et les tensions qui peuvent exister entre chercheurs, lorsqu'ils sont mis en position d'experts face aux décideurs, aux personnels œuvrant dans le domaine de la santé, aux nombreux débats publics organisés. Elle a permis en outre de découvrir la difficulté, et même le désarroi, des responsables politiques placés devant les conséquences multiples - dont certaines peu prévisibles - de leurs décisions sur tous les champs de l'activité humaine... au point de se retrancher derrière le paravent de la légitimité scientifique (par ailleurs remise en question à la suite de certains débats). Et que dire des personnels de santé qui ont dû bouleverser leurs environnements de travail, leurs pratiques, et faire preuve de beaucoup de créativité et d'engagement pour faire face aux décisions prises par des autorités, aux besoins nouveaux émergents, à des ressources (matérielles, financières, humaines) insuffisantes, tardives ou plus suffisamment adaptées.

Les mutations de notre société avaient été mises en évidence lors du colloque international organisé par la Revue internationale d'éducation de Sèvres (RIES) et du numéro thématique qui a suivi ("Réformer l'éducation ", n 83, 2019). La revue a donc jugé utile et même nécessaire de concevoir un numéro sur l'état de la recherche en éducation dans les différentes régions du monde, dans le but d'observer son impact sur les systèmes éducatifs et leurs acteurs (décideurs et praticiens, et en dernier ressort les élèves), et en conséquence, grâce à la posture comparative qui la caractérise, de dégager à quelles conditions la recherche en éducation pourrait avoir un impact à la fois plus fort mais aussi plus pertinent sur la prise de décision et l'action menée sur le terrain. Entre le moment où nous envisagions le présent numéro et celui de sa rédaction est intervenue la crise de la Covid; celle-ci a conforté chez les auteurs l'importance de la question posée.

Pour articuler les informations apportées par les contributions issues de différentes régions du monde, et donc de contextes socioculturels et politiques très divers, le concept de pouvoir nous semble être un point d'entrée particulièrement pertinent. Selon des auteurs tels que Crozier et Friedberg, le pouvoir est la capacité d'action d'un acteur (personne, groupe ou organisation) dans ses relations 
avec autrui ainsi que l'interaction entre des acteurs n'ayant pas la même possibilité d'action. Dans le cas présent, nous sommes bien en présence de trois types d'acteurs: les chercheurs, les décideurs et les acteurs de terrain (non seulement les chefs d'établissement et les enseignants, mais aussi les autres membres du personnel éducatif, sans oublier les élèves et leurs parents praticiens). Tous trois appartiennent à des groupes et à des organisations spécifiques. Il s'agit donc pour nous: 1) de commencer par circonscrire où est le pouvoir des uns et des autres; 2) d'examiner comment ces différents pouvoirs entrent en tension ou se rencontrent dans des relations duelles ou duales; 3) enfin de tenter de dégager les conditions qui permettraient aux différents pouvoirs (celui de la recherche et du savoir, celui de la décision et de la politique, celui de l'action et de la contextualisation) de créer de l'intelligence collective ou collaborative pour permettre aux systèmes éducatifs de répondre aux besoins d'éducation, de faire face aux mutations sociétales et de s'améliorer.

\section{OÙ EST LE POUVOIR DE LA RECHERCHE EN ÉDUCATION?}

\section{Les logiques de recherche et leur pouvoir}

La recherche est un processus qui consiste à produire des connaissances nouvelles par une démarche rigoureuse validée par la communauté scientifique. Le savoir scientifique a un double pouvoir: il permet d'affronter la réalité en étant mieux armé qu'en se basant sur des préjugés (le savoir scientifique est l'objet d'une démarche de légitimation, même si celle-ci reste toujours provisoire; les préjugés ne le sont pas); et paradoxalement, la production de connaissances scientifiques nouvelles élargit notre conscience de ce que nous ne savons pas et nous invite au doute. Ce double pouvoir donne au chercheur un pouvoir d'analyse critique quant à l'utilisation des connaissances qui est faite dans d'autres champs.

Au sens fondamental du terme, la recherche produit trois types de connaissances emboîtées: elle établit des faits objectivables, énonçables, mesurables; sur cette base, elle recherche des relations récurrentes entre des catégories de faits pour expliquer un phénomène; elle tente d'articuler un ensemble de relations récurrentes en un schème (une «bonne forme " ou Gestalt) apportant une compréhension plus pertinente de l'objet étudié que les schèmes antérieurs. Quand il s'agit de recherche dont l'objet est l'éducation, on retrouve ces trois niveaux de recherche. Dans le premier cas, il s'agit d'établir des observatoires ou des bases de données sur l'éducation, ce qui suppose l'utilisation de méthodologies descriptives et édumétriques validées par la communauté scientifique; ces recherches, souvent initiées par une ou plusieurs équipes de chercheurs, sont ensuite reprises par des instances territoriales, nationales (comme la direction de l'évaluation, de la prospective et de la performance - DEPP en France) ou internationales (PISA en est l'exemple le plus connu). Dans le second cas, les chercheurs tentent d'établir des relations entre des catégories de faits observés (exemple: entre des dispositifs didactiques et des performances évaluées), soit à l'aide d'outils corrélationnels, soit au moyen de 
dispositifs expérimentaux ou quasi expérimentaux de comparaison. Dans le troisième cas, les chercheurs sont préoccupés de comprendre un phénomène éducatif dans toute sa complexité et non en le réduisant comme dans le cas précédent. Deux grands types de démarches s'offrent alors au chercheur: 1) le schème compréhensif nouveau est postulé au départ sur la base d'une articulation originale de diverses connaissances scientifiques, voire expérientielles, antérieures et il est ensuite validé ou invalidé par un nouveau recueil de données ou grâce à des données disponibles et traitées à cet effet; 2) le schème compréhensif est construit par une démarche plus inductive sur la base de phases successives de recueil de données et de confrontation aux connaissances antérieures et à des hypothèses nouvelles.

Formalisé dans le monde anglo-saxon après la Seconde Guerre mondiale, aux États-Unis principalement, sous l'impulsion du behaviorisme et de méthodologues bien connus internationalement (comme Skinner, Thurstone, Spearman, Thorndike, Stanley et Campbell, Rosenshine, Gage, Wittrock ${ }^{1} .$. ), ce courant toujours bien vivant est souvent dénommé "la recherche sur l'éducation ", désignant par là que le chercheur regarde en surplomb et le plus objectivement possible l'objet éducatif étudié (la psychologie et la pédagogie expérimentale, et actuellement les neurosciences appartiennent à ce courant). Dans ce dossier, l'article de Thierry Karsenti et Maurice Tardif sur la recherche au Québec décrit bien comment celle-ci prend historiquement son origine dans ce courant, à la suite des décisions prises par le « Rapport Parent » dans les années 1960, à la base du système éducatif québécois actuel.

La réflexion des chercheurs sur la nature de l'objet étudié, l'éducation, a mis en évidence le caractère multidimensionnel de l'acte éducatif: 1) une dimension épistémique, car il s'agit de connaissances à faire construire et d'un certain rapport à la connaissance à inculquer (débat actuel brûlant avec la montée en puissance des réseaux sociaux, des fake news, des "conflits de vérité à l'école ", comme le montre le numéro 77 de la RIES (2018); 2) une dimension praxéologique, car la connaissance et le rapport à la connaissance se construisent dans l'action entre des "élèves » et des " enseignants» (ou tout autre médiateur, comme d'autres élèves, des personnes externes sans oublier les parents), au sein d'une organisation avec ses niveaux de décision, et dans un environnement fait de ressources et de contraintes; 3) une dimension axiologique, car sont porteurs de valeurs tant le choix des connaissances (RIES n ${ }^{\circ} 73$, "Ce que l'école enseigne à tous », 2016), que le rapport à la connaissance, la posture relationnelle entre les acteurs, la structure et la circulation de la décision à travers l'organisation, les opportunités présentes ou au contraire absentes dans l'environnement.

À l'image des sciences dites "dures »dont les connaissances servent différents champs d'application et où, en retour, la recherche appliquée devient de plus en plus le lieu de découverte de connaissances fondamentales (y compris en mathématiques), les sciences de l'éducation développent de plus en plus des recherches pour l'éducation, dont la finalité première est d'améliorer l'éducation grâce à un travail de recherche collaborative avec les acteurs de terrain et qui, en

1. N'oublions que les deux Handbook of Research on Teaching, édités sur le plan scientifique en 1963 par Gage et en 1986 par Wittrock, ont été pendant longtemps les bibles des chercheurs en éducation dans le monde entier, du moins pour ceux qui pouvaient lire l'anglais. 
retour, permet de valider les modalités de transfert de certaines connaissances scientifiques dans un ou plusieurs contextes spécifiques ou/et de produire des connaissances scientifiques en termes de schémas compréhensifs et systémiques de différents contextes d'action. L'article de Xu Mengjie sur la recherche menée " par » et « avec » les enseignants d'un district de Shanghai illustre ce mouvement.

Le processus de légitimation des recherches sur l'éducation a surtout concerné la validité scientifique et la fiabilité. La légitimation des recherches pour l'éducation a plutôt mis l'accent sur la validité écologique (le rapport d'adéquation au contexte), l'efficacité (le rapport entre effets observés et attendus) et l'efficience (le rapport entre les effets observés et les investissements déployés par les acteurs). La réflexivité étant au cœur de telles recherches collaboratives, elle a mis en évidence que l'action pouvait être efficace et efficiente sans être pertinente, par exemple en étant inéquitable ou en éteignant la curiosité au fil de la scolarité, ou encore en créant des apprenants dépendants, etc. Éducation et société sont deux objets d'étude étroitement reliés: l'école (formelle, mais aussi non formelle et informelle) est-elle (doit-elle être) le reflet de la société dans laquelle elle baigne² ? L'école contribue-t-elle (devrait-elle contribuer) à construire la société de demain? Derrière ces questions se pose celle de la pertinence de la prise de décision et de l'action en éducation: d'une part, le rapport entre les effets attendus de la décision et les effets qu'il aurait fallu attendre; d'autre part, entre les effets réellement observés de l'action et les effets qu'il aurait fallu pouvoir observer. C'est-à-dire la question des valeurs: les décideurs et les acteurs de terrain ne peuvent y échapper, car ne pas (vouloir) se poser la question est déjà un choix implicite de valeurs. En serait-il autrement pour le chercheur en éducation? La contribution de Khalaf Al'Abri et Omer Hamad montre comment les chercheurs en éducation dans les pays arabes sont confrontés à cette question brûlante: pour des motifs politiques, sociaux ou culturels, philosophiques ou religieux, ils ne peuvent aborder avec des méthodes scientifiques, analytiques ou critiques des objets comme le tribalisme en éducation, la mixité à l'école et bien d'autres. À l'inverse, selon Sally Power et Chris Taylor, le Pays de Galles est un cadre idéal pour développer un "écosystème de preuves en matière éducative " permettant d'« éclairer et questionner la compréhension des processus et résultats éducatifs». Le questionnement des valeurs et la posture de la preuve ne sont pas incompatibles; conjointement, elles caractérisent la forme d'engagement du chercheur en éducation. Quand il s'agit de poser le problème des valeurs, de mettre au jour les valeurs sousjacentes à un processus éducatif, voire à tout un système éducatif, nous ne sommes plus simplement dans une logique épistémique de recherche sur l'éducation, ni dans une logique praxéologique de la recherche pour l'action, mais dans une logique axiologique de la recherche de la valeur de l'éducation projetée ou mise en œuvre. Cette préoccupation a été au fil des siècles (voir RIES n ${ }^{\circ} 79$, "Figures de l'éducation ", 2018) une question fondamentale de la philosophie et de l'histoire de l'éducation. En apportant des preuves empiriques, la sociologie de l'éducation a mis en évidence l'importance du déterminisme social dans et par l'éducation, telle qu'elle fonctionne dans sa forme scolaire actuelle. Cependant, trop peu de recherches empiriques ayant le souci d'apporter la preuve se sont penchées sur la question politique: quelles

2. Dans sa treizième chronique, Alain Bouvier formule la question de cette façon: « Un pays n'a-t-il pas l'école qu'il mérite? » Voir: https://www.mlfmonde.org 
valeurs (ou contre-valeurs) sont développées par tel environnement éducatif, par tel dispositif pédagogique... et donc finalement par tel système éducatif. Question essentielle pour toute personne ayant charge d'éduquer, à plus forte raison pour les décideurs responsables du devenir de la société.

\section{Des tensions au sein même de la communauté scientifique}

Le pouvoir de la recherche est donc avant tout lié à sa capacité à produire de la connaissance, dont la preuve est objet de validation par la communauté scientifique. Mais ce pouvoir est l'objet de tensions à l'intérieur du monde lui-même des chercheurs, tensions mises en lumière par les auteurs de ce numéro. Ainsi, décrite par Pons et Rey, la diversité en France des lieux de production, liée à la diversité des disciplines, de leurs épistémologies et de leurs méthodologies, entraîne des «cloisonnements ", voire une «balkanisation » et des hiérarchisations dans les représentations des unes et des autres, nuisant ainsi aux tentatives d'" institutionnalisation de nouvelles interfaces ». C'est nettement moins le cas au Québec. Karsenti et Tardif expliquent comment la forte demande de scolarisation au Québec au $\mathrm{xx}^{\mathrm{e}}$ siècle a entraîné la création des facultés d'éducation à travers le territoire pour répondre à la demande et fonder la formation et le développement des personnels d'éducation sur la base de connaissances scientifiques. Et c'est au sein de ces facultés qu'ont été créées des équipes de recherche; des fonds importants ont été débloqués pour financer non seulement leurs travaux, mais aussi la formation de jeunes chercheurs. Ce même souci d'allier recherche en éducation et développement professionnel des enseignants se retrouve en Suède. Elisabet Nihlfors et Staffan Selander nous expliquent que l'éducation est «fondée sur des bases scientifiques et une expérience approuvée par la recherche »: au cours de leur formation, les enseignants sont donc placés dans un « environnement de recherche active ».

Une autre tension se matérialise à travers le slogan bien connu «publish or perish ». La recherche en éducation, dans la plupart des pays, est assurée par des universitaires qui ont une double, voire triple mission (l'enseignement, la recherche, et même des charges de gestion et de service). Malgré la lourdeur parfois excessive des charges d'enseignement pour les jeunes enseignants surtout et des charges de gestion pour les moins jeunes, le développement de la carrière des enseignantschercheurs repose essentiellement sur le curriculum scientifique, dans la plupart des pays. Le besoin de publier entraîne de nombreuses dérives. Parlant des pays arabes (mais le constat est bien plus étendu), Al'Abri et Hamad constatent que, pour les chercheurs, le besoin du nombre de projets et de publications est bien plus important que la production de connaissances nouvelles. Cela entraîne un recours à la réplication de dispositifs de recherche menés ailleurs en privilégiant les recherches quantitatives, plus facilement publiables dans certaines revues anglophones, et en multipliant les articles sur le même corpus de données. Ce besoin de publier n'est pas étranger à la profusion de revues scientifiques ou prétendues telles ${ }^{3}$.

3. Voir, par exemple: De Ketele J.-M. (2019). «La publication scientifique en sciences de l'éducation et de la formation : état des lieux, dangers et perspectives ». Les Dossiers des sciences de l'éducation, $\mathrm{n}^{\circ}$ 41, p. 43-59. 
Sally Power et Chris Taylor soulignent aussi le danger, dans les pays plus petits ou au PIB plus faible, comme le Pays de Galles, de voir les chercheurs les plus qualifiés être recrutés par les structures de recherche de pays voisins dominants (en l'occurrence l'Angleterre), offrant des environnements scientifiques plus riches, des financements plus importants, pour mener de gros projets et bénéficier de voies de reconnaissance internationale plus assurées. Comme le soulignent Masengesho Kamuzinzi et Safari Kambanda, cette tendance à l'évaporation des chercheurs se constate encore davantage dans des pays africains, aux dépens de leur pays d'origine et au profit des pays occidentaux ou d'institutions internationales.

\section{OÙ EST LE POUVOIR DE LA DÉCISION EN ÉDUCATION?}

Répondre de façon nuancée à cette question aurait supposé d'identifier qui, dans un territoire donné: 1) prescrit et contrôle les finalités du système éducatif (Denis Meuret parle de "récit mobilisateur ", voir RIES n 83, 2020); 2) prescrit et contrôle le curriculum (contenu, supports didactiques, modalités, évaluation); 3) prescrit et contrôle les aspects organisationnels et réglementaires; 4) attribue et nomme les personnels éducatifs aux différents postes à fournir; 5) dispose des moyens financiers et les attribue à qui et pour quels postes et fonctions (dont la recherche)... Si répondre de façon précise à de telles questions pourrait être l'objet de travaux complémentaires, la réponse à certaines questions touche en revanche celle des rapports entre le pouvoir des décideurs et celui des chercheurs, selon leur nature et les lieux où il s'exerce.

Pour notre propos, on peut se demander où se trouve vraiment le pouvoir de décision des grandes orientations du système éducatif d'un pays et dans quelle mesure les connaissances issues de la recherche y contribuent. À l'examen des articles de ce dossier, il semble que le pouvoir de décision se situe bien au-delà des ministères de l'Éducation, dépende bien plus du contexte et des enjeux sociopolitiques que du désir de construire un récit mobilisateur avec les acteurs de l'éducation et l'éclairage des chercheurs. Plusieurs articles illustrent cette thèse.

Un premier exemple est celui de la Russie. Darya Loyola et Svetlana Dudko montrent la volonté des responsables politiques russes d'accorder la priorité à l'unification de territoires nombreux et divers, qui couvrent deux continents. L'enjeu politique (éviter l'éclatement du pays) est énorme. Une arme pragmatique est trouvée: imposer un " examen d'État unifié » entre le secondaire et l'enseignement supérieur. En évitant l'éclatement de l'éducation des jeunes, les autorités politiques espèrent contribuer dans la durée à l'unification du pays. Les institutions de recherche, nationales et régionales, seront mobilisées pour construire et légitimer les outils.

Le contexte sociopolitique du Mexique est différent. Carlos Ornelas explique que si à certaines époques les ministres et hauts fonctionnaires de l'Éducation se sont entourés de personnalités influentes issues du monde de la recherche, «les hommes politiques avancent à l'instinct et le désir de se maintenir au pouvoir prime sur tout autre désir ». Par ailleurs, au fil du temps, on voit les grandes organisations internationales exercer de plus en plus de pressions. C'est notamment le cas de l'OCDE (dans le cadre de l'accord de libre-échange avec les États-Unis et 
le Canada), de la Banque mondiale (l'aide financière est conditionnée à certaines réformes), l'Unesco (en être membre donne une certaine légitimité), l'Unicef (qui oserait ne pas signer la Charte des droits de l'enfant?). Mais finalement, le souci de rester au pouvoir amène à diverses alliances et compromissions qui ne permettent pas aux tentatives de réformes qui se succèdent de produire leurs effets.

Pour l'Afrique, Masengesho Kamuzinzi et Safari Kambanda soulignent que les acteurs politiques sont très «vulnérables face aux nouvelles réformes éducatives [...] qui sont pour la plupart du temps initiées à l'échelle supranationale et souvent légitimées comme des engagements partagés par tous les pays concernés ». Elles sont d'ailleurs le plus souvent " des énormes opportunités de financement » de la Banque mondiale, du Fonds monétaire international, de la Banque africaine de développement et des coopérations bilatérales qui ont leurs propres intérêts et experts. Si l'Afrique possède des intellectuels brillants, les uns sont marqués par une sorte de "syndrome d'exportation » de modèles de pensée créés ailleurs pour comprendre les phénomènes africains, les autres s'opposent à tout ce qui vient des pays colonisateurs. À ce propos, on (re)lira avec intérêt la tentative d'analyse d'Alioune Sall sur le devenir potentiel de l'Afrique (RIES nº 83, "Réformer l'éducation »).

Avec la Suède, on se trouve dans une culture scandinave marquée par le protestantisme. Les processus de décision revêtent une tout autre structure que celles exposées ci-dessus. Comme en Finlande, la plupart des décisions se situent au niveau des municipalités. Aux autorités centrales, il revient de fixer et d'anticiper les grandes orientations en "se fondant sur des bases scientifiques et une expérience approuvée par la recherche». Aux municipalités de les opérationnaliser et de les contextualiser grâce à des acteurs formés à la recherche et bénéficiant de l'encadrement de chercheurs, comme nous le verrons plus loin.

Dans la section suivante, nous verrons que dans des pays réputés fortement centralisateurs, une prise de conscience de l'importance de la recherche comme dispositif de production des connaissances en collaboration avec les acteurs de terrain semble faire son chemin.

\section{OÙ EST LE POUVOIR DE L'ACTION EN ÉDUCATION?}

Au fil du temps, la recherche en éducation a accumulé de nombreux résultats de recherche. L'analyse critique de 800 méta-analyses effectuée par John Hattie en $2008^{4}$ a montré que le fait le plus probant à en retirer est le pouvoir des acteurs sur le terrain: pas d'amélioration ou de transformation (pertinente ou non) des systèmes éducatifs sans l'adhésion, l'engagement et la capacité de ceux-ci. Comme le montre Carlos Ornelas, les syndicats d'enseignants mexicains ont été suffisamment puissants, à une époque de leur histoire, pour prendre un pouvoir suffisant afin d'obtenir par chantage des avantages qui ont corrompu et dégradé l'ensemble du système éducatif et sociétal. Le pouvoir d'action peut agir positivement comme négativement. 
Les exemples décrits dans ce numéro tendent à montrer que l'implication conjointe des chercheurs et des acteurs de terrain augmente positivement le pouvoir de l'action, d'autant plus qu'elle est soutenue politiquement et financièrement. L'article d'Elisabet Nihlfors et Staffan Selander est à cet égard particulièrement éloquent. Ainsi, une municipalité suédoise de soixante établissements scolaires a créé un service de recherche et de développement ayant pour responsable un enseignant titulaire d'un doctorat, travaillant en coopération avec deux universités, permettant aux enseignants de s'impliquer dans des équipes mixtes de recherche sur des sujets pertinents. Les structures mises en place diffèrent d'une municipalité à l'autre, mais Halverson et Kelly (2017), cités dans leur article par ces auteurs, montrent que ces projets de $\mathrm{R} \& \mathrm{D}$ fournissent les moyens d'un développement professionnel continu au niveau de l'école et de la communauté.

De telles initiatives à cet égard tendent à se multiplier ces dernières années, même dans des pays reconnus par la prégnance du pouvoir central ou/et la dispersion de la recherche. Ainsi, Xavier Pons et Olivier Rey signalent qu'en France, des structures territoriales d'enseignement (les académies) ont mis en place des CARDIE ("cellules académiques de recherche, innovation et expérimentation») qui, au-delà du soutien et du conseil, encouragent les initiatives locales des enseignants et la collaboration avec des chercheurs. À un niveau plus large que les académies, les LéA («lieux d'éducation associés») sont des réseaux d'enseignants et de praticiens visant à mettre en place un pilotage stratégique et scientifique, mais ceux-ci manquent de soutien de la part de l'État central.

Renforcer conjointement le pouvoir de l'action et le pouvoir de la recherche correspond bien à une tendance forte actuelle au Québec. Les «centres de service scolaires» (qui ont remplacé depuis le 15 juin 2020 les " commissions scolaires») y sont des instances de gouvernance locale regroupant les établissements d'enseignement. Thierry Karsenti et Maurice Tardif expliquent que plusieurs facteurs vont pousser les chercheurs en éducation à collaborer davantage avec elles: 1) comme cela a déjà été mentionné, les facultés d'éducation ont été créées pour augmenter quantitativement et qualitativement le développement professionnel des enseignants; 2) les chercheurs en éducation ont pris de plus en plus conscience que se fonder sur les seules données probantes des recherches sur l'éducation, synthétisées dans les méta-analyses, ne suffisait pas à améliorer les pratiques de terrain et qu'il fallait conduire et valoriser des types de recherches contextualisées avec les acteurs de terrain; 3) des organismes de financement important ont été créés, tout particulièrement le FRQSC (Fonds de recherche société et culture du Québec) recommandant et rendant même obligatoires non seulement l'intégration de praticiens dans les équipes de recherche, mais aussi des actions de transfert des résultats de recherche dans les réseaux d'éducation.

Les exemples ci-dessus montrent l'importance du local dans la collaboration entre praticiens et laboratoires de recherche. Cela signifierait-il que le partenariat entre acteurs de terrain et chercheurs est impossible dans un État centralisé particulièrement fort? L'exemple de l'article chinois de Xu Mengjie mérite notre réflexion, même si ce qui est rapporté ne concerne qu'un district de la seule région de Shanghai (particulièrement florissante sur les plans économique et culturel). Les acteurs de la politique locale mise en place sont: le Bureau régional de l'éducation, représentant 
le ministère de l'Éducation nationale dont la directive prône « la participation active des écoles et des enseignants à l'expérimentation en matière de réforme éducative »; l'Institut d'éducation, qui assure la formation et des services pour le développement professionnel, notamment en collationnant et diffusant les résultats des recherches nationales et internationales en éducation; les chefs d'établissement et les enseignants, encouragés à lire beaucoup, à expérimenter des pratiques pédagogiques, à partager leurs expériences lors de sessions de formation. Ainsi décrit, il s'agit bien d'un pilotage " top down » de type prescriptif, qui a très vite fait apparaître les carences tant des enseignants sur le terrain que des chercheurs de l'Institut d'éducation chargés de les accompagner. Pour y remédier, des mesures fortes ont été prises: des enseignants ont été mis en position de chercheurs et les chercheurs en position d'enseignants. Par ailleurs, un établissement public particulièrement médiocre a été transformé en établissement pilote et les conventions suivantes ont été contractualisées: 1) les nouveaux professeurs de l'Institut d'éducation doivent d'abord enseigner dans cet établissement pilote; 2) les enseignants expérimentés du district et de l'Institut d'éducation enseignent tous les trois à cinq ans dans l'établissement pilote pendant un an; 3) des enseignants qualifiés dans les écoles sont sélectionnés pour participer à la recherche sur l'établissement pilote et en diffuser les résultats. Au-delà du fait que l'établissement pilote est devenu particulièrement performant, Xu Mengjie souligne un double impact de ce pilotage local basé sur la double compétence d'enseignant et de chercheur: l'élévation des niveaux de motivation et de compétence des personnes; la création d'un écosystème régional performant au bout de plusieurs cycles (on est bien dans un système politique planifié).

Dans un contexte culturel et sociopolitique très différent, le CRIFPE (Centre de recherche interuniversitaire sur la formation et la profession enseignante), dans lequel Thierry Karsenti et Maurice Tardif assument des responsabilités de direction, contribue aussi à créer des écosystèmes régionaux au sein du Québec grâce à ses 124 chercheurs attachés aux différentes universités régionales, et même dans d'autres régions du monde comme l'Afrique grâce à 41 chercheurs internationaux, mais aussi et surtout par l'implication d'acteurs de terrain dans les équipes de recherche.

Les exemples rapportés ci-dessus ont un point commun, un partenariat praticiens-chercheurs, prenant des formes différentes selon les cultures (scandinave marquée par le protestantisme, latine teintée de rationalisme bureaucratique, anglosaxonne empreinte de pragmatisme, sinisée ancrée dans le confucianisme, latino-américaine imprégnée de la pensée de Paulo Freire, musulmane attachée à la religion). Construire un écosystème de développement basé sur la preuve par l'action et la recherche est sans doute aussi quelque peu différent selon la taille du pays et son insertion dans un territoire plus vaste: le Pays de Galles souffre par exemple de la position dominante de son puissant voisin et voit ses chercheurs s'exporter.

Il serait injuste de terminer cette section en laissant penser que la situation de certaines régions est telle que leurs acteurs de terrain sont complètement livrés à eux-mêmes alors qu'ils vivent des conditions extrêmement difficiles. Ainsi, dans les pays d'Afrique et comme le soulignent Masengesho Kamuzinzi et Safari Kambanda, les institutions internationales, en position de force, négocient leur soutien directement avec les plus hauts responsables qui font le nécessaire pour satisfaire aux 
conditionnalités exigées... sans que les acteurs de terrain ne se sentent concernés. Cependant, disent-ils, il existe dans ces pays des intellectuels de qualité. Deux organismes, l'Agence universitaire de la francophonie (AUF) et l'Agence française de développement (AFD) en ont pris conscience et ont uni leurs forces pour coordonner et financer le projet Apprendre. Il s'agit d'une plateforme d'expertise visant à créer dans les pays francophones (dix pays en 2017, vingt-six en 2019) des «écosystèmes apprenants »: des chercheurs africains sont associés aux enseignants et aux chefs d'établissement pour améliorer les pratiques de terrain par et avec la recherche, produire des connaissances et des savoir-agir contextualisés. Il s'agit là d'une initiative importante à suivre... en espérant que ces chercheurs pourront en tirer des publications scientifiques et des revues pour les publier (le problème est loin d'être résolu).

\section{Créer une intelligence COLLABORATIVE : VERS UNE ALLIANCE DES POUVOIRS DE LA RECHERCHE, DE LA DÉCISION ET DE L’ACTION?}

La recherche en éducation comme pratique réflexive argumentée remonte à un passé lointain et a généré de grandes "figures de l'éducation dans le monde » (thème du $\mathrm{n}^{\circ} 79$ de la RIES, 2018), très inspirantes mais ayant beaucoup de difficultés à pénétrer le système formel. La recherche en éducation en tant que discipline scientifique reconnue, beaucoup plus récente, est le fruit d'un long cheminement. Les historiens s'accordent à dire qu'en créant le premier laboratoire de psychologie expérimentale à Leipzig en 1875, Wundt a inspiré les premières initiatives: en 1883, un cours de «science de l'éducation » à la Sorbonne (Durkheim en a été titulaire); en 1903, création de la Society of Educational Research aux États-Unis par J.M. Rice, d'origine allemande et ayant connu Wundt; création de laboratoires de "pédagogie expérimentale » à Genève, en Belgique et en France au début du xx ${ }^{e}$ siècle; création en 1958 de l'AIPELF (Association internationale de pédagogie expérimentale de langue française) avec Gaston Mialaret comme premier président; naissance en 1977 de l'AMSE (Association mondiale des sciences de l'éducation). Mais il s'agissait là d'initiatives de chercheurs. Les États ont mis plus de temps à leur accorder une place institutionnelle autonome et, dans bien des pays elles sont encore inféodées à des facultés des lettres, contrairement aux pays de culture anglo-saxonne où les facultés d'éducation ont une existence propre. Il n'est donc pas étonnant que, surtout dans les pays où elle ne bénéficie pas encore d'une véritable reconnaissance, la recherche en éducation n'ait guère d'influence sur la prise de décision politique ni sur les pratiques de terrain. Les prises de conscience actuelles, générées par les mutations sociétales et les bouleversements dans le monde de l'éducation provoqués par la pandémie de la Covid-19, incitent à croire que, comme dans le monde de la santé, une alliance des différents pouvoirs (de la recherche, de la décision et de l'action) est plus que jamais nécessaire. Des opportunités se présentent, mais des freins subsistent. À partir des contributions des auteurs de ce dossier, mais aussi des travaux recensés dans l'intéressante bibliographie qui l'accompagne, conçue par Bernadette Plumelle, nous tentons ci-dessous de les identifier. 


\section{Quels freins faut-il desserrer?}

Des freins existent d'abord chez les chercheurs eux-mêmes, lorsqu'ils restent enfermés dans leur discipline d'origine et contribuent ainsi à composer une mosaïque de recherches et de connaissances juxtaposées sur un objet pourtant complexe et nécessitant une articulation systémique. Cet enfermement est accentué par l'exigence du publish or perish qui privilégie, à des fins carriéristes, la multiplication d'un certain type de recherches, essentiellement des recherches quantitatives sur l'éducation dont les connaissances restent trop générales pour servir efficacement la prise de décision politique et l'action sur le terrain. À cela s'ajoute souvent le syndrome de la tour d'ivoire, qui se manifeste par des résistances telles que la peur d'une approche trop utilitariste, ou par des réflexes, comme « ce n'est pas mon problème » ou « il n'y a qu'à appliquer» (voir par exemple l'analyse de Duru-Bellat, 20195).

Comme cela apparaît à maintes reprises dans les contributions de ce numéro, les responsables politiques ont aussi des intérêts carriéristes (être réélus et rester au pouvoir), prennent des décisions sur des bases plus idéologiques que scientifiquement argumentées ou se servent de la légitimation scientifique pour justifier après coup des décisions déjà prises. Hormis dans quelques pays où existe une continuité du pouvoir politique, le temps politique est un temps court, alors que les effets d'une politique et d'une action éducative relèvent du temps long. Comme le soulignait le no 83 de la RIES « Réformer l'éducation » (2020), plus de 150 réformes ont été relevées ces dernières années; elles se succèdent sans que les précédentes aient pu être évaluées et que l'on ait pu espérer en voir les effets attendus. L'absence d'une politique à long terme de financement des recherches en éducation (et pas simplement des plans qui se font et se défont) ou la difficulté des décideurs à passer des commandes pertinentes de recherche (Cytermann, 2018) sont sans doute le signe le plus manifeste du peu d'intérêt ou de la capacité à fonder la politique et l'action éducative sur des bases solides. Mais le frein le plus grave mis en évidence dans ce dossier réside dans l'interdiction, explicite ou implicite, faite aux chercheurs d'aborder certains aspects de l'éducation pour des raisons politiques, culturelles, philosophiques et religieuses.

À ces freins s'ajoutent ceux que l'on peut relever chez les acteurs du terrain où s'opère le processus éducatif. Ceux-ci estiment ne pas avoir accès aux résultats de la recherche en éducation faute de supports de communication adaptés: ils ne sont pas « lisibles »; ils sont trop " généraux »; ils ne sont pas " utiles », car ils ne tiennent pas compte des contraintes de leur contexte; ils demandent "trop d'efforts » et supposent des temps de formation qui ne sont pas prévus.

\section{Quelles opportunités saisir?}

Même lorsqu'il est isolé (voir l'article de Kamuzinzi et Kambada sur les pays africains, par exemple), tout chercheur a maintenant conscience que, malgré sa jeunesse, la recherche en éducation a produit une masse énorme de connaissances scientifiques, accessibles, solidement établies et qui ont été synthétisées dans des méta-analyses de plus en plus nombreuses (la Review of Educational

5. Les références des auteurs cités dans cette section se trouvent dans la bibliographie établie par Bernadette Plumelle annexée au dossier. 
Research ${ }^{6}$ 'est spécialisée dans ce type de publication) et même désormais dans des méga-analyses comme celle de Hattie (2008, op. cit.). Comme dans d'autres domaines (la santé, par exemple), cela a provoqué dans un premier temps un élan pour associer recherche et prise de décision, à travers ce que l'on appelle l'evidence-based policy (connue aussi dans le monde francophone sous le nom d'" enseignement explicite ", à la suite de l'ouvrage canadien de Gauthier et al., $\left.2013^{7}\right)$. Avec le recul et sans en sous-estimer l'importance, de nombreux chercheurs ont pris conscience que de telles connaissances solidement établies sur le plan scientifique ne sont pas transférables comme telles en contexte. En effet, de telles connaissances sont "toutes choses restant égales par ailleurs ", ce qui n'est jamais le cas dans la réalité. Un chercheur comme Bryk a publié avec des collègues une série d'articles (consulter la bibliographie jointe au dossier et son article dans le $\mathrm{n}^{\circ} 83$ de la RIES), dans lesquels il insiste sur la nécessité de mener et de financer des recherches (Bryk est président de la Fondation Carnegie pour l'amélioration de l'enseignement) sur la manière de transférer les résultats des recherches sur l'éducation (une forme d'intelligibilité) sur le terrain de la pratique éducative, c'està-dire dans une multiplicité de contextes, tous différents car de nombreux facteurs y interagissent. Cela nécessite de recourir à des recherches pour l'éducation, impliquant une autre forme d'intelligibilité grâce à la collaboration des chercheurs et des acteurs concernés par l'objet d'étude: pratiques fondées sur les preuves (evidence-based practice) et preuves fondées sur la pratique (practice-based evidence) sont en étroite interaction et produisent de l'intelligence collaborative.

Les responsables politiques se sentent de plus en plus obligés de légitimer les décisions qu'ils prennent, tant ils sont «soumis à la question " par les médias, tant les organisations internationales, malgré l'absence de pouvoir légal sur l'éducation, ont des moyens de pression de plus en plus forts: en termes d'évaluations internationales (on a montré le pouvoir important que jouait sur les responsables politiques la communication de leurs classements internationaux, voir le $\mathrm{n}^{\circ} 54$ de la RIES en 2010, "Palmarès et classements en éducation »); mais aussi en termes de financement de réformes dans certains pays; ou encore en termes de financement de mobilités et de projets pour des chercheurs, des responsables, des enseignants, des formateurs, des étudiants; et plus fondamentalement pour certaines d'entre elles (comme l'Unicef et l'Unesco) en termes de droits humains et sociétaux fondamentaux. Toutes ces initiatives aident à sortir des œillères nationales et à prendre conscience que d'autres possibles existent.

Comme le montrent les articles de ce dossier, les conditions d'exercice de la mission d'enseignement et d'éducation varient très fortement d'une région du monde à l'autre. Et cependant, les acteurs de terrain (chefs d'établissement, enseignants, autres personnels d'éducation, y compris les parents) déclarent être confrontés à des problèmes de plus en plus complexes. Malgré le confort apporté par l'exercice en solitaire de leur métier (auquel sont attachés particulièrement les enseignants et leurs syndicats... et parfois même les enseignants-chercheurs), les problèmes rencontrés

6. Cette excellente revue est malheureusement d'un coût très élevé pour beaucoup de centres de documentation et n'est pas en accès libre.

7. Voir Gauthier C., Bissonnette S. et Richard M. (2013). Enseignement explicite et réussite des élèves. Louvainla-Neuve : De Boeck 
sont si complexes qu'une prise de conscience émerge sur la nécessité d'un fonctionnement plus solidaire (constat effectué dans le $n^{\circ} 83$ de la RIES 2020). La crise de la Covid-19 n'est pas étrangère à la montée en puissance de celle-ci. De plus en plus aussi, la forme scolaire (faite de juxtaposition bureaucratique d'espaces, de contenus et de temps, comparable à une " usine ") s'avère inadaptée face aux problèmes posés et une dynamique créative se développe chez les acteurs de terrain.

\section{Les voies d'une intelligence collaborative}

Des opportunités sont ouvertes ou existent, encore faut-il les mobiliser. Les états des lieux dressés dans ce numéro nous conduisent à penser qu'il faut exploiter davantage tous les espaces qui créent de l'intelligence collaborative en étant des espaces de médiation.

Les associations scientifiques internationales sont des espaces de médiation qui permettent aux chercheurs de sortir de leurs cloisonnements, lorsqu'elles sont pluridisciplinaires et ouvertes aux décideurs et aux praticiens qui y viennent avec des études de cas soumises à discussion pour articuler les expertises des uns et des autres. Des associations telles que l'American Educational Research Association (AERA), l'Association pour le développement des méthodologies d'évaluation en éducation (ADMEE), l'Association internationale de pédagogie universitaire (AIPU) (et d'autres) jouent déjà ce rôle.

Les gros laboratoires de recherches pluridisciplinaires, ayant l'éducation et la formation comme objet d'étude, obtiennent plus facilement des financements régionaux, nationaux et internationaux, et peuvent placer plusieurs chercheurs aux compétences complémentaires sur des projets importants. Sous la responsabilité de personnalités telles que Thierry Karsenti et Maurice Tardif, le CRIFPE joue ce rôle au Québec, et même au-delà avec ses 41 chercheurs internationaux, d'autant plus qu'il associe dans ses recherches de nombreux acteurs de terrain qui ont l'occasion de se former à et par la recherche, et de participer au transfert des connaissances produites activement sur le terrain. Des initiatives de ce type existent ou sont en germe dans certains pays; il est indispensable de les soutenir.

Dans de nombreux pays, les centres de formation des enseignants ont souvent des fonctionnements trop solitaires. Même si elle n'est pas transférable comme telle dans d'autres cultures, l'expérience décrite à Shanghai montre bien l'importance d'établir des relations étroites entre formateurs, chercheurs, chefs d'établissement scolaire, enseignants. Les centres de formation sont en principe bien placés pour être des «centres de veille scientifique »: l'implication de leurs étudiants, futurs enseignants, dans le suivi des résultats de la recherche en éducation et dans la constitution de synthèses avec l'aide de leurs formateurs et de documentalistes, devrait être une composante importante de leur formation; les veilles produites par les institutions de formation devraient pouvoir être disponibles dans les établissements scolaires où les étudiants effectuent leurs stages et constituer des supports utiles à la réflexivité et dans lesquels les étudiants, les maîtres de stage et les tuteurs académiques puisent pour fonder et justifier leurs analyses. L'expérience de Shanghai nous indique aussi que les centres de formation ne doivent pas s'enfermer dans la seule formation initiale mais doivent s'impliquer dans les établissements 
scolaires: non seulement le développement professionnel des acteurs de l'établissement scolaire le nécessite, mais aussi et tout autant celui des formateurs des institutions de formation. Le projet Apprendre, conduit dans des pays en développement et soutenu par des organismes de la francophonie, l'a bien compris. De telles initiatives devraient inciter les responsables politiques nationaux à rendre possibles de tels espaces de travail collaboratif.

«Recherche, politique et pratiques: espaces, acteurs et supports de médiation »: ce $n^{\circ} 201$ de la Revue française de pédagogie (2017), coordonné par Sylvain Doussot et Jean-Yves Rochex, souligne l'importance, pour les responsables politiques, de disposer également de "passeurs ", ce qui demande des compétences que n'ont pas nécessairement les chercheurs. Heureusement, des initiatives se développent de plus en plus parmi lesquelles: 1) les veilles au niveau national (par exemple, les veilles de l'IFE en France) ou international (comme celles du centre de ressources et d'ingénierie documentaires (CRID) de France Éducation International, qui alimentent aussi cette revue) ; 2) les revues d'interface au niveau national (comme Vie pédagogique au Québec ou Administration et éducation en France) ou au niveau international comme la présente Revue internationale d'éducation de Sèvres à visée comparatiste; 3 ) la rubrique éducation des grands journaux nationaux qui organisent de plus en plus des échanges avec leurs confrères étrangers ou voient leurs articles repris et synthétisés dans des organes de presse comme Courrier international; 4) les think tanks ou les " conférences de consensus». Des organes et des passeurs existent. Encore faut-il qu'il y ait " passage » et que le passage soit fréquenté par ceux qui détiennent le pouvoir de décision; encore faut-il que ces derniers prennent le temps non seulement de s'informer, mais d'en débattre (ce qui suppose une présence active; une simple lecture de documents ou de rapports faits par des conseillers ne suffit pas). Et pourquoi pas imaginer des "Davos de l'éducation » ou toute autre forme d'activité de développement professionnel des responsables politiques eux-mêmes?

Les politiques de financement de la recherche en éducation sont souvent le reflet du degré de connaissance que les responsables politiques ont des résultats de celle-ci; elles sont aussi le signe du degré de reconnaissance qu'ils manifestent envers les chercheurs, comme on peut le voir à travers les articles de ce dossier. Rapportées dans la bibliographie de ce numéro, certaines initiatives sont stimulantes. Ainsi, Magrath, Aslam et Johnson font état d'un consensus croissant sur la nécessité de penser la recherche sur les résultats d'apprentissage dans un cadre plus large, comme c'est le cas du programme de trente projets financés par le ministère britannique du développement international (DFID) et le Conseil de recherche économique et sociale (ESRC), programme qui vient compléter le programme de recherche en cours Raising Learning Outcomes in Education Systems.

Pensé dans le prolongement du dossier "Réformer l'éducation » (RIES $\mathrm{n}^{\circ} 83,2020$ ), ce numéro nous indique que le monde de la recherche, celui de la prise de décision politique et celui de l'action ont chacun leur pouvoir, malheureusement exercé de façon trop solitaire. C'est au contraire par l'alliance de ces pouvoirs en termes de complémentarité, non de supériorité ni de confusion mais en créant de l'intelligence collaborative, que les systèmes éducatifs peuvent s'améliorer et se transformer progressivement pour affronter les défis sociétaux qui nous sont posés actuellement sous des formes diverses selon les contextes. 